\title{
Digital education reform for improving interaction between students and instructors
}

Qiansong Deng, Yuanjie Li, Lixin Zheng

Qiansong Deng, Yuanjie Li, Lixin Zheng, "Digital education reform for improving interaction between students and instructors," Proc. SPIE 10452, 14th Conference on Education and Training in Optics and Photonics: ETOP 2017, 104525Q (16 August 2017); doi: 10.1117/12.2269871

SPIE Event: 14th Conference on Education and Training in Optics and Photonics, ETOP 2017, 2017, Hangzhou, China 


\title{
Digital education reform for improving interaction between students
}

\section{and instructors}

\author{
Qiansong Deng, Yuanjie Li and Lixin Zheng \\ School of Optical and Electronic Information, Huazhong University of Science and Technology, \\ Wuhan 430074, China, Email: dqs961000@ hust.edu.cn
}

\begin{abstract}
Nowadays it is difficult to attract undergraduate students' interesting to put sufficient time to learn major courses in China, which are too hard for them to quick grasp and fully understanding. Here we report a digital education reform for improving interactions between students and instructors, in which we transform the abstract, obscure and boring knowledge, such as physical, mathematical, electronic or optical concepts into direct and dynamic 3-D model and flash. Therefore, this method can convert theoretical concepts into easy understanding pictures. Our several years' experience shows that this education mode can make students' willing to think and practice, then it is helpful for attracting their learning interests. Most students benefit from this education mode which can greatly enhance their understanding abilities.
\end{abstract}

Keywords: Scientific and engineering pictures; interaction between instructor and student; digital education reform

\section{PREFACE}

Theory is the guideline of action. In order to implement the college innovative education reform, it is indispensable to figure out the theory of innovative education at first. According to R.K. Sawyer's study, there is no everlasting definition for innovation which always evolves with time and has strong ties with society and history. [1] In addition, innovation can be interpreted completely different depending on the culture and knowledge background the interpreters have. After the mid-21st century, with the rapid development of knowledge society, the raising of study science and the social culture evolution of innovative research, knowledge-based and practice-driven innovation with specificity of different areas has gained increasingly widespread attentions [2,3,4] At present, the well-accepted characters of education in top universities include:

(1) courses that can reflect the latest research achievement in their respective fields.

(2) research-based teaching model and high challenge content of courses.

(3) integrated experimental education system, multi-layers education for innovation and entrepreneurship.

(4) high-level teacher teams to undertake the teaching tasks including courses for junior students.

(5) evaluation of teachers based on teaching, research and other aspects.

(6) integrated evaluation system for students referring to performance of their course study and so on.

(7) comprehensive quality monitoring system on both class and off-class.

14th Conference on Education and Training in Optics and Photonics: ETOP 2017, edited by Xu Liu,

Xi-Cheng Zhang, Proc. of SPIE Vol. 10452, 104525Q - @ 2017 ICO, IEEE, OSA, SPIE

CCC code: $0277-786 X / 17 / \$ 18 \cdot$ doi: $10.1117 / 12.2269871$

Proc. of SPIE Vol. 10452 104525Q-1 
(8) recruitment standard based on both comprehensive qualities and specialties.

(9) high ratios of international teachers and student, of staff and students and of large class teaching and small class teaching.

(10) reasonable knock-out system for students.

The techniques of computer numerical computation, simulation and thought judgement bring powerful new motivation and vital technical support for modern education reform. By applying this reform together with combining the scientific thought culture and modern information computing methods to teaching, it is possible for us to awake students' willingness to study, think, practice and finally they can make succeed and enjoy themselves. Furthermore, with the help of simulation and virtual design, we successfully deliver the abstract, obscure and boring concepts in physics, math, electrics and optics via direct and dynamic 3-D model or flash, which can help students easily understand and grasp the basic idea of the abstract concepts. In short, the reform includes all well-recognized characters of education in top universities mentioned above.

\section{BASIC MEASURES FOR DIGITAL EDUCATION REFORM BASED ON THE INTERACTION OF STUDENTS AND TEACHERS}

The so-called digital teaching refers to the teaching mode that seamlessly combines the modern computer programming technology, Internet technology, fundamental course teaching and ideological education. The specific approach is to use digital technology to integrate three quantitative descriptions (i.e. algebraic description of characters and symbols, geometrical description of graphs and description of final values) with computer science and numerical simulation, and to add the description of dynamic and high dimensionality, the function of interaction, rendering, and thinking judgment, in order to constitute the most integrated modern scientific quantitative description system. The introduction of digital technology to teaching would induce the great change of the traditional teaching. Specific measures are divided into the following three aspects:

\subsection{Off-line classroom teaching}

Taking 'Applied Partial Differential Equations' as an example, the off-line classroom teaching for this course was initiated by Professor Li Yuanjie, a national famous teacher. The specific procedures include [5]:

(1) The book 'Applied Partial Differential Equations' includes five topics, six ideas, ten equations and ten methods.

(2) Providing students with various, content-rich teaching resources for students to freely choose.

(3) Introducing modern educational technology into the teaching process to quantitatively simulate physical phenomena, and turn the abstract physical model (differential equation) into a dynamic physical phenomenon.

(4) Teachers bring their own research experience and results into the teaching process which would inspire students to explore the topics more by themselves and thus help them to form the ability of innovation.

(5) Design specific teaching procedure and training tasks. After introducing scientific thinking and research methods, energy modeling, mathematical iterative method, the computer graphics method, all those should be applied in the homework, essays and examinations. 
(6) According to the different teaching contents and the characteristics of research methods, the teaching processes should flexibly include the teachers' abstracts of teaching and students' autonomous learning, problem research and writing at anywhere necessary.

(7) Take research learning into the final score.

(8) Using the Chinese cultural and scientific ideas for the mathematical formula and knowledge of the high induction is another highlight of this teaching mode.

\section{2. off-line extension after class}

Taking the course of 'applying partial differential equation' as an example, after the class, the teacher requires the students to use the "scientific calculation and simulation" software, which is developed by students themselves independently, as the mathematical tool to finish the homework after class, and select the best work to make into a book. By holding exhibition of college students "scientific computing and simulation", in the school of OEI, HUST in 2014, we realize perfect combination of "Internet + " information technology and teaching. The education reform has great impact on the development of students' ability of surviving and develop in the workplace, independent studying and innovation, and using modern information technology. The exhibition is shown in Figure 1 and Figure 2 (a) - (d).

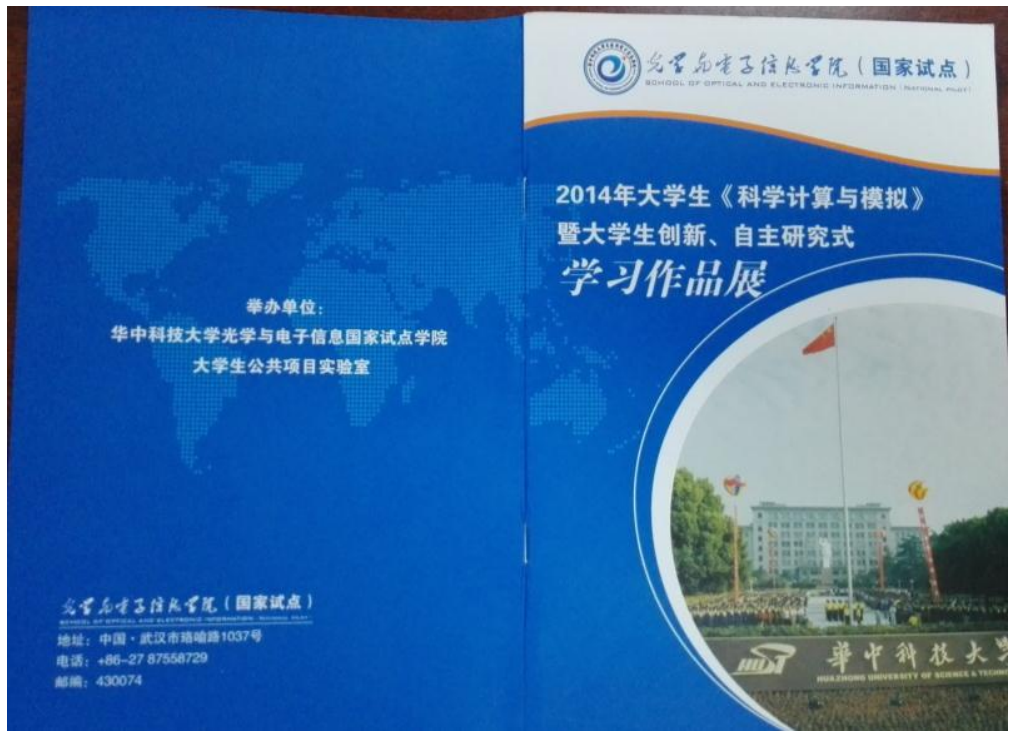

Figure 1. 《Scientific Computing and Simulation》 College students' innovative and automatic research album

a

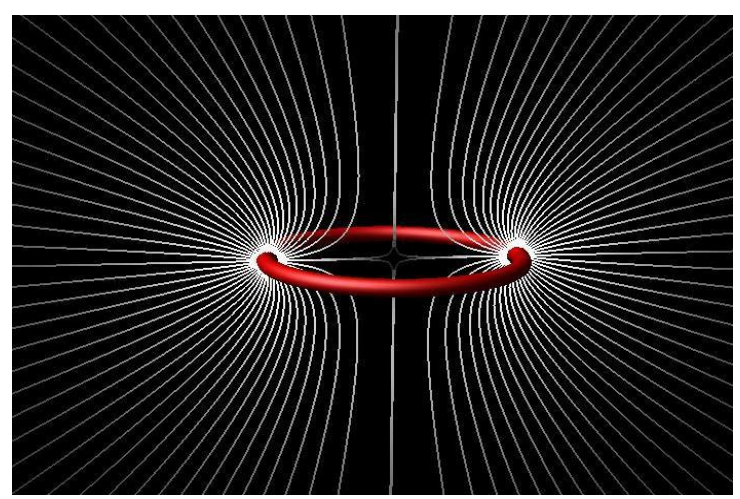

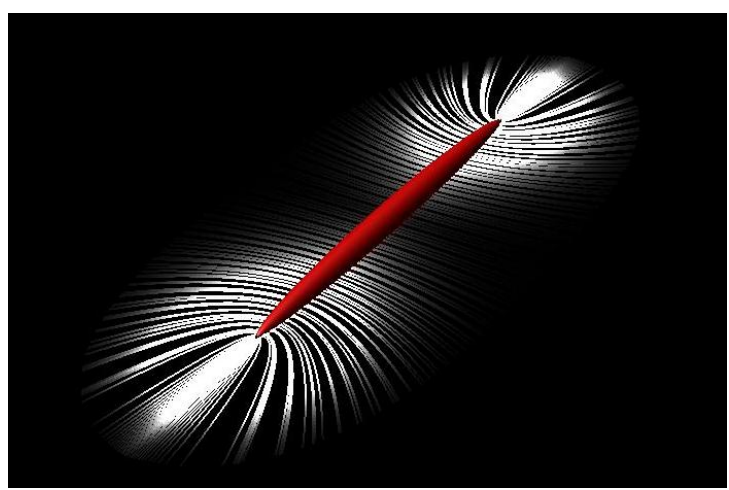



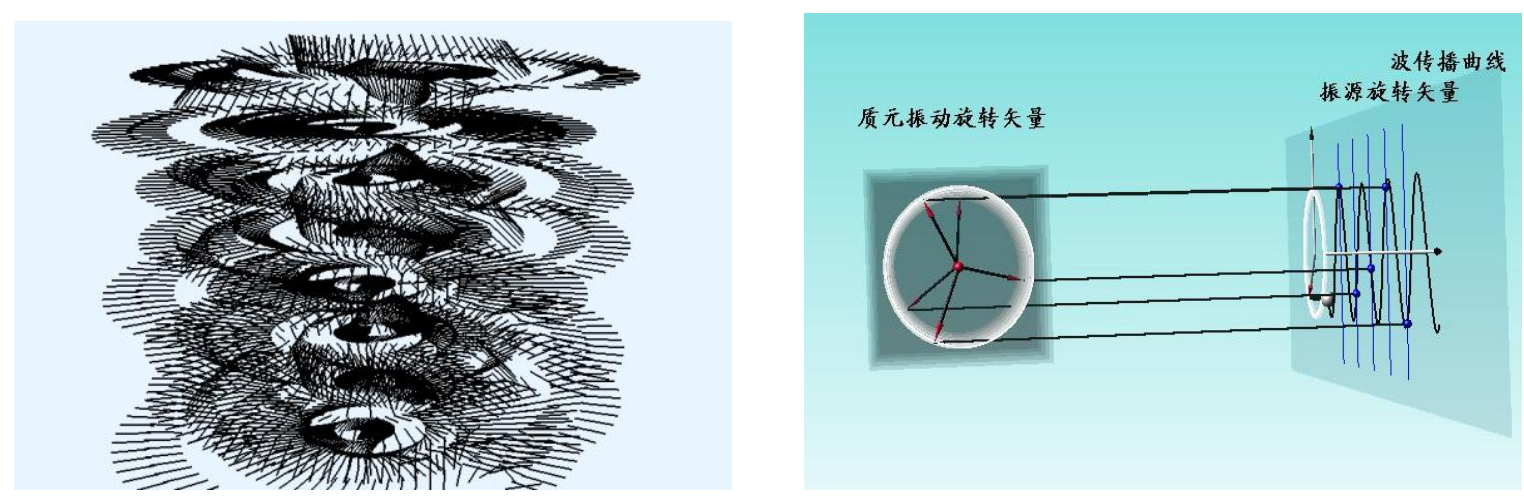

Figure 2. (a)the field of uniformly charged ring, (b) surrounding electricity field of metal tips, (c) rectangular waveguide in cylinder figure, (d) relation between vibration and wave.

\subsection{Web-based courses}

In order to help students to thorough fully understand the knowledge they have learned in the classroom, the teaching group makes full use of the modern (mobile) Internet technology to produce the Chinese Digital Mock Course (CDMOOC) for the classroom teaching. The various forms such as small class of important content make students enjoy mobile phone reading interaction mode anywhere and anytime without restriction which provides the great flexibility for students to learn. As shown in Figure 3, we provide the two-dimensional codes for Chinese digital Mu-class.
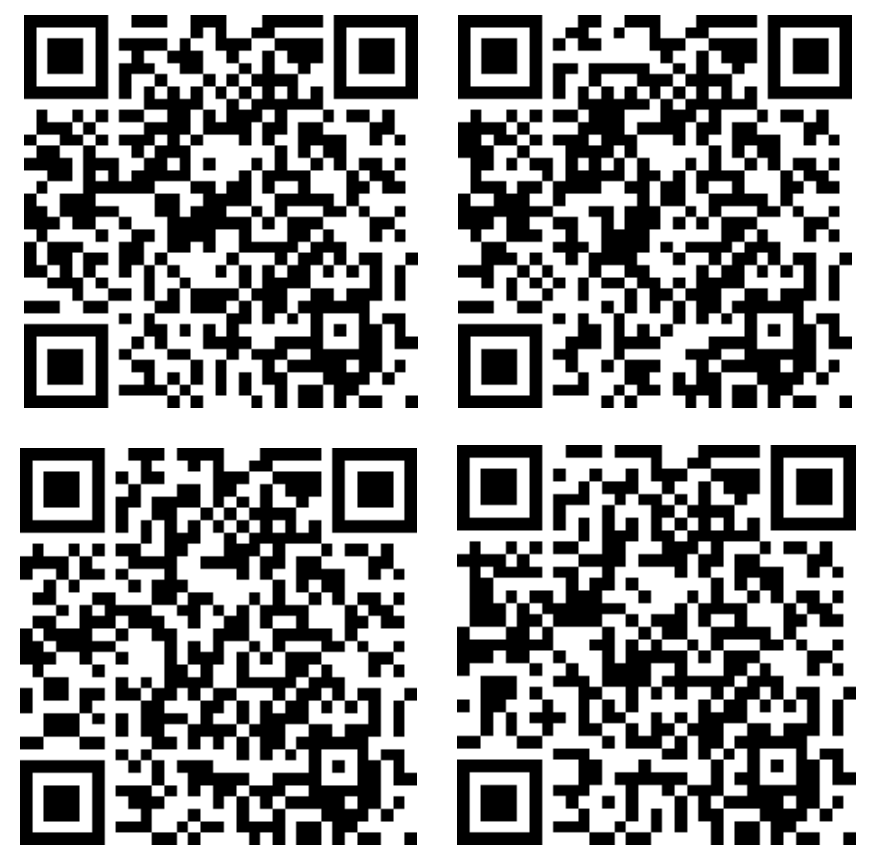

Figure 3. 2D code of CDMOOC 


\section{MAIN FEATURES OF THE DIGITAL EDUCATION REFORM}

The digital education reform based on the teacher-student interaction transforms from teaching fundamental knowledge only to focusing on fundamental knowledge together with scientific thought and scientific research methods. With the combination of teachers' conducting and students' automatic research, we gradually shift our concentration from knowledge teaching to inspire students learning under the teachers' instruction. The main features include:

(1) Introducing the modern information education techniques, especially digital and internet techniques into the classroom; by applying the modern information techniques' platform to teaching so at to realize the realization and visualization of specialized fundamental theories.

(2) According to the core idea of "Separate the class into several teams", 3 or 4 students form a team as the unit of class and undertake fundamental courses design training on the selected projects using the simulation software; the group members should follow the procedure: decompose work-literature review-design the schemes-simulation-research results-oral presentation; Through implementing the innovative education mode of "Community of Study", we successfully complete the research study based on courses, bring out students' passion of study and improve their abilities of utilizing knowledge, cooperation and management to some extent.

(3) Constructing the formative evaluation system. The final grade of courses is mainly evaluated based on daily homework $(10 \%)$, class performance $(10 \%)$, experimental projects with computers (20\%), final exam (60\%). Students cannot take the final exam if their first three grades are less than 24 score. The formative evaluation system plays an active role in evaluating the study performance and solving the common problem that students always prefer to work hard just before the exam rather than pay attention to the daily course study.

\section{EFFECT OF THE GENERALIZATION AND APPLICATION OF THE DIGITAL EDUCATION REFORM}

As the digital curriculum reform emphasizes on knowledge, thinking and method, it fully combines the analytical, numerical calculation, and Chinese thinking logic, which contains strong Chinese features. This digital curriculum reform is especially suitable for innovative education training mode and shows great potential to promote the replacement of exam-oriented education and pure knowledge education in Chinese colleges and universities. The digital curriculum reform has implemented for 5 years and has been applied to over 10,000 students in two universities (Huazhong University of Science and Technology and China (East China) University of Petroleum). The result reveals that the new digital curriculum is warmly welcomed by the students. The exhibitions of students' work have been held for three times and the digital curriculum reform exhibits the remarkable advances over the traditional ones mainly in the following areas:by applying this digital curriculum reform, the junior undergraduates can establish mathematical models for physical phenomena, and published academic papers. Furthermore, these students continuously perform very well in software design, Challenge Cup competition and graduation thesis

when they enter the senior year. This reform completely alters the previous teaching mode that only established and well-accepted knowledge should be taught during the classes and has proven to greatly enhance the ability of solving practical problems and innovation.

(2) Taking the 《application of partial differential equations》 as an example, the new teaching mode attracts a number of students to participate in the reform. Many students take this as a good opportunity to improve their abilities, 
show their personality and test their perseverance. The students participating in this teaching reform have common comments about this: "The most challenging experimental class", "pain and happiness!". Many students use this experience to prove their ability of scientific research when they apply for work position and graduation program either at home or abroad.

(3) In order to further generalize the digital curriculum reform results, the National Experimental College of OEI, Huazhong University of Science and Technology, held the China Digital MOOC show and press conference in December 3, 2016, in Wuhan. During the conference, a number of works independently made by the undergraduate students from school of optical and electronic information, who have taken the digital reform course 《applied partial differential equations》 have been displayed.

The displayed works have attracted extensive attention and given high praise by the leaders of the higher education sector, experts and scholars attended the show. A number of medias such as Hubei TV, Chutian Metropolis Daily, Wuhan Evening News,Phoenix and other relevant medias attended the conference and some of the leadership, guests and teachers and students were interviewed on site.It is worth to mention that the Hubei public news channel broadcast up to 2 minutes and 30 seconds of live interviews video in the evening of the date the news conference held.

\section{CONCLUSION}

Based on the interaction between teachers and students, taking full advantage of modern information technology , using the mode of combining the online and offline class and relying on the college public project laboratory based on the National Experimental Institute of OEI, the digital teaching reform tries to explore how to efficiently train undergraduate students with high creative skills from five aspects related to the modern innovation education - learning, thinking, action, result, interesting, namely how to "learn and think, think and practice".

The results show that the digital teaching model based on teacher-student interaction can help students to develop the ability including how to survive and grow in the workplace, self-learning and innovation and using modern information technology.

The teaching reform would effectively promote the reform of personnel training system and teaching content as well. Furthermore, the reform would significantly improve the students' practical ability, research potential and innovative consciousness. The undergraduate innovation and teaching system have established now, thus it is worth to further generalize it.

Nevertheless, due to the limited number of people engaged in the project, the reform limits to a few teachers and courses and demands for further generalization. Meanwhile, the practical activities of the course group need to be further deepened and refined from the theoretical aspects.

\section{REFERENCES}

[1] Sawyer, R. K., [The Science of Human Innovation], East China Normal University Press, Shanghai, 105-247 (2013).

[2] Zhang, Qiong., Chen, You. qing., "Knowledge - based practical ability and its educational implications and training strategies," Educational Development Research. Papers 24(1), 41-46 (2010). 
[3] Qian, Gui. qing., Liu, Wen. li., [An Introduction to Innovative Education], Beijing Normal University Press, Beijing, 8-14 (2012).

[4] Zhang, Qiong., "Knowledge application and innovation ability cultivation," Higher Education Research. Papers 3(11), 62-67 (2016).

[5] Xu, Xiao. dong., Feng, Xiang. dong., "Research and practice of research - oriented teaching model for undergraduates of science and engineering," Chinese University Teaching. Papers 11(3), 9-14 (2008). 